\title{
Superior Septal Approach for Mitral Valve Surgery Incase of Better Left Atrial Exposure
}

\author{
Kenan Abdurrahman Kara*1 ${ }^{*}$ and Fatma Ferda Kartufan ${ }^{2}$ \\ ${ }^{1}$ Department of Cardiovascular Surgery, Yeditepe University Hospital, Turkey \\ ${ }^{2}$ Department of Anesthesia and Intensive Care, Yeditepe University Hospital, Turkey
}

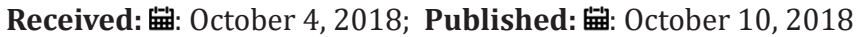

*Corresponding author: Kenan Abdurrahman Kara, Department of Cardiovascular Surgery, Yeditepe University Hospital, Turkey

Abstract

There are various options to access the left atrium in surgical interventions performed on the mitral valve. Transapical approach is used to implant transcatheter valves for mitral bioprosthesis failure (valve in valve implantation) and implantation of artificial neo-chordae to correct mitral regurgitation [1,2]. It is also subsequently accessed through a standard left atriotomy incision from both left and right side of the atrium, superior or dome approach, right atriotomy (trans septal, superior septal, inferior trans septal, and extended trans septal approaches), trans aortic approach, and finally through the left ventriculotomy. Excellent visualization of the operative area is the key for a successful mitral valve surgery. Various clinical conditions and the body habitus of the patient do affect good surgical exposure, and the surgeon should be aware of all these approaches so that he can choose the right approach to a given patient. In this study, we offered superior septal approach as a second option particularly in cases where adequate exposure in small left atrium was hard to achieve with conventional left atriotomy.

Patients and Methods: 20 patients underwent mitral valve replacement operation using cardiopulmonary bypass (CPB) by a single surgeon between March 2017 - November 2017 at the Hisar Intercontinental Hospital. 20 patients (6 males, 14 females; mean age: 53.8) underwent MVR, and 6 of them had additional CABG, Tricuspit De Vega, and left atrial thrombus extraction operation using superior septal approach (Table 1).

Results: There were 14 women and 6 men. None of the patients had ischemic mitral valve regurgitation, 12 had mitral valve prolapse, and 8 had rheumatic heart disease. The mitral valve was replaced in all patients. 6 patients bioprosthesis valve and mechanical replacement in 14. There were no perioperative complications associated with the atriotomies, i.e. no bleeding, no atrioventricular nodal dysfunction, and no sinus node dysfunction. The extended vertical transatrial septal approach provides good mitral valve exposure without inherent complications.

Conclusion: In surgeries performed with the superior septal approach which we used in our study, it provides a good exposure in cases where the left atrium is particularly small. It is evident that using consecutive right atrium, fossa ovalis and left atrial dome incisions and closing these incisions in the appropriate anatomical position instead of reaching the mitral valve with a single left atriotomy increase the cross-clamp time. However, considering the loss of time and risk of complication in cases where adequate exposure cannot be achieved with the conventional left atriotomy, we think that this is an approach that should be in the repertoire of the surgeon as a quite good option.

Keywords: Mitral valve surgery; Superior septal approach; Exposure for mitral valve surgery

\section{Introduction}

The aortic (AV), tricuspid and pulmonary valves are repaired or replaced through a single approach into their corresponding chamber. The MV can be accessed through multiple anatomic approaches for repair or replacement. The MV is also the valve farthest from surgeon's view. Excellent visualization of the operative area is the key for a successful mitral valve surgery. Previous heart operation, being obese or thin, etiology of mitral valve disease and associated small size of the left atrium are factors affecting surgical vision. Many characteristics lead the surgeon to consider various incisions for the correct approach.

Median sternotomy, mini sternotomy, right anterior thoracotomy or mini thoracotomy are some of the options [3].
After incision is started with one of these options, several incisions like left atrium, right aterium and trans septal approach, through ascending aorta and left ventricle as well, are made in heart cavity to provide the best exposure in order to reach the mitral valve. When these characteristics are evaluated together, the surgeon has to make a decision for the most correct exposure and the shortest clamp time.

Furthermore, cardiac pathologies may change the anatomy of the left atrium and mitral valve. Calcifications around the mitral valve ring and hypertrophic right ventricle may contribute to the narrowness of the left atrium. Additionally, scars and adhesions lead to mobility loss in the mitral valve and surrounding tissues and make surgery more difficult. 
Conventional left atriotomy provides access to the mitral valve by opening of the left atrium with a $3-5 \mathrm{~cm}$ incision from the interatrial groove. This is the most common approach. In rheumatic mitral stenosis and in chronic severe mitral regurgitation, the LA is generally quite large, and this can provide excellent exposure with left atriotomy. The atriotomy can be extended on the superior aspect below the right pulmonary artery and also on the inferior aspect $[4,5]$. This is generally a reliable technique for most patients with chronic MR and can be reproduced without much difficulty.

The approach with left atriotomy in mitral valve surgery is the most frequently used method. With this incision, a simple and smooth valve surgery exposure can be ensured. Optimal mitral valve operation requires adequate exposure without impairment of atrial physiology. However, some atriums can be challenging for valve surgery performed with the conventionel left atriotomy. Small left atrium, advanced calcifications and thrombus in the left atrium are the first to come to mind. Left atrial ecarteur used to increase the inadequate exposure in such cases may lead to some complications from dysfunction of the sinus node and atrioventricular node function due to excessive traction to tears, and as a result, mortality. The superior septal approach, which includes the incision of the right atrium, opening of fossa ovalis and opening of the left atrium, including the left atrial roof in an upward direction provides a quite good exposure without using left atrial ecarteur. This approach is allowing a clear and wide exposure of the mitral valve with a minimal risk of tearing of the left atrial wall [6-8]. We used this incision in patients who underwent MVR with median sternotomy as well as 4 patients who underwent right anterior mini thoracotomy.

\section{Surgical Technique}

Superior trans septal approach. After insertion of one cannula in the inferior vena cava and one cannula in the superior vena cava, an incision was made in the right atrium in parallel to the atrioventricular groove and was expanded to the superior pole of the atrial septum. After obtaining cold blood cardioplegia, a septal incision including the fossa ovalis was performed, and the incision was continued to the left atrium. At the end of the mitral valve surgery, the roof of the left atrium and the septal incision were closed with a 3-0 prolene suture. Then, the air was cleared from the left side and the aortic clamp was opened. The right atriotomy was closed with a 4-0 prolene suture.

Sinus node dysfunction is one of the main concerns following MVS with the STS approach. Berdajs [2003] claimed that possible injury of the sinus node artery during the STS approach is related to ischemia of the sinus node and results in sinus node dysfunction. In another hypothesis, Lukac stated that the incision in the STS approach creates a barrier to impulses from the sinus node, which is located on the posterior of the right atrium, preventing them from reaching the other side of the heart.

\section{Patients and Methods}

20 patients underwent mitral valve replacement operation using cardiopulmonary bypass (CPB) by a single surgeon between
March 2017 - November 2017 at the Hisar Intercontinental Hospital. 20 patients ( 6 males, 14 females; mean age: 53.8 ) underwent MVR, and 6 of them had additional CABG, Tricuspit De Vega, and left atrial thrombus extraction operation using superior septal approach (Table 1).

Table 1: Operative and postoperative data of patients with Superior Septal Approach.

\begin{tabular}{|c|c|}
\hline Patient number & 20 \\
\hline Age (Years) & 53,8 \\
\hline Male & 6 \\
\hline Female & 14 \\
\hline RAMT & 4 \\
\hline Sternotomy & 16 \\
\hline Cross Clamp Time & 67,1 \\
\hline Drainage & $550 \mathrm{ml}$ \\
\hline ICU Day & 2,2 \\
\hline Hospital Day & 6,9 \\
\hline Additional Procedures & 6 \\
\hline Tricuspit DeVega & 3 \\
\hline CABG & 5 \\
\hline LATE & 1 \\
\hline
\end{tabular}

RAMT: Right Anterior Mini-Thoracotomy; LATE: Left Atrial Thrombus Extraction

Surgery was performed through a median sternotomy in 16 patients and right anterior minthoracotomy in 4 patients with cardiopulmonary bypass establishing selective venous cannulation through vena cava superior and vena cava inferior and ascending aortic cannulation. Blood cardioplegia was used in the initial and intermittent cold cardioplegia was used thereafter. Hypothermic cardiopulmonary bypass was started with rectal temperature lowered to $30^{\circ} \mathrm{C}$ or $32^{\circ} \mathrm{C}$. Tapes were snared around the inferior and superior venae cavae. The right atrium was opened longitudinally along the anterior segment. The atrial septum was incised vertically through the fossa ovale. The right atriotomy was prolonged superiorly into the right coronary fossa between the right atrial appendage and the atrioventricular sulcus to join the superior end of the septal incision. Consecutively, the roof of the left atrium was incised. The left atriotomy was extended to the left over, at a distance from the aortic root. Continuous stay sutures were placed. A retractor was used for additional exposure. A clear and wide exposure of the mitral valve apparatus was completed without dislocation or distortion. The operation on the valve could proceed using either repair or replacement. Concurrent cardiac operation was carried out before or after mitral valve operation. Atriotomies were closed using 3-0 monofilament running sutures. The superior left atrial incision was closed, then the septal incision starting at the lower end upward and the right atrial incision were closed. The cross clamp occluding the aorta was then removed. An intravenous second-generation cephalosporin antibiotic was administered intraoperatively and for 48 hours postoperatively for prophylaxis against infection. 
Patients were followed up for a mean of 2.2 days in intensive care. They were then taken into the general ward and discharged after a mean of 6.9 days. The patients were assessed in terms of operative mortality, cross clamp time, length of intensive care stay and hospitalization, post-operative complications, and reoperation.

\section{Statistical Analysis}

No statistical tests were used. Data were expressed as a mean \pm standard deviation.

\section{Results}

There were 14 women and 6 men. None of the patients had ischemic mitral valve regurgitation, 12 had mitral valve prolapse, and 8 had rheumatic heart disease. The mitral valve was replaced in all patients. 6 patients bioprosthesis valve and mechanical replacement in 14 . There were no perioperative complications associated with the atriotomies, i.e. no bleeding, no atrioventricular nodal dysfunction, and no sinus node dysfunction. The extended vertical transatrial septal approach provides good mitral valve exposure without inherent complications.

With the superior-septal approach exposure of the mitral valve apparatus has been satisfactory without the need for forceful retraction. In all cases, only stay sutures were used. It was believed that mitral valve operation was made easier and shorter. Mean aortic cross-clamping time for mitral valve replacement was 67.1 minutes. Repair of atriotomy was not associated with bleeding or postoperative dehiscence such as an atrial septal defect. The septal segment was repaired by closing the edge of the fossa ovale. There was no atrioventricular nodal dysfunction, and no pace-maker usage.

\section{Discussion}

There are several complications that may occur in routine mitral valve surgery. Cerebral emboli, injury to circumflex coronary artery, A-Vgrove disruption, leftventricular rupture duringremoval of failed prosthesis, perivalvular leak after prosthetic valve implantation, and uncontrolled bleeding during redo cardiac surgery from caval perforations are the most common complications. It is evident that cross clamp time is markedly increased with the superior septal approach. However, the clear exposure it provides technically facilitates the intervention to the mitral valve especially in patients with a smaller left atrium diameter. Therefore, it minimizes the risk of the above-mentioned complications. In terms of surgical time, it takes a long time to open the right atrium, fossa ovalis and left atrial dome and to close all of them in the appropriate anatomic plan following the operation but in patients with small left atrium who undergo left atriotomy, it relatively decreases the surgical time in selected cases when considering the time loss that may be experienced while dealing with complications that may occur due to excessive traction in the ecarteur, which do not happen with the superior septal approach. That's why even the standard left atriotomy gives adequate exposure of the mitral valve most of the times, the surgeon should also consider the superior septal approach as a second incision option where left atriotomy cannot provide adequate exposure [9-12].

The superior approach is also called as the dome approach and is exposed between the SVC on the right side and the Asendan Aorta on the left side. The left atrial wall is clearly seen below the right pulmonary artery above and the aortic root below. The incision can be extended towards superior pulmonary vein on the left side and on the right side behind the superior vena cava. Injury to the sinoatrial (SA) node should be avoided as well as to the SA nodal artery during the retraction and incision into the left atrium. Another issue to consider when performing an incision is not to let the incision be too close to the aortic root. Attention should be paid to the complications such as tear or bleeding that may occur due to applying sutures close to the aortic wall when closing the incision line. Instead of placing a retractor to retract the friable left atrial wall, multiple pledgeted stay sutures can be used for retraction [1317].

\section{Conclusion}

There are many studies on the comparison of the classical left atrial groove exposure and superior septal approach in the literature. However, this is not a completely fair comparison in our opinion. It is without dispute that the conventional method provides an adequate exposure and the cross-clamp time is less when compared with the patients undergoing routine mitral valve surgery. However, we come across tear due to excessive traction use (VCI, left atrial dome) or post-operative arrhythmia and blocks due to sinus node and AV node damage when ecarteur is used in patients with a particularly small left atrium. As a result of these complications, cross clamp time increases considerably. When superior septal approach is used in such cases, it ensures a smooth valve surgery without a need for ecarteur with the help of a wide and good exposure and stay sutures, which are the mostly used ones, without causing the above complications. In surgeries performed with the classical method, using ecarteur due to inadequate exposure can lead to tears due to excessive traction, and this may progress to mortality. Instead of comparing the two techniques in this study, we wanted to point out that an excellent surgical exposure and outcome without complications can be achieved with the superior septal approach in suitable cases without using left atrium ecarteur.

In our opinion, superior septal approach to the mitral valve is safe and reproducible. It gives excellent exposure of the mitral valve under all circumstances without a significant increase in crossclamp or bypass time, postoperative arrhythmia, heart block/ pacemaker rate, or bleeding. In conclusion, we have reported an approach to the mitral valve that is associated with adequate exposure and no inherent complications.

\section{Scientific Responsibility Statement}

The authors declare that they are responsible for the article's scientific content including study design, data collection, analysis and interpretation, writing, some of the main line, or all of the 
preparation and scientific review of the contents and approval of the final version of the article.

\section{Animal and Human Rights Statement}

All procedures performed in this study were in accordance with the ethical standards of the institutional and/or national research committee and with the 1964 Helsinki declaration and its later amendments or comparable ethical standards. No animal or human studies were carried out by the authors for this article.

\section{References}

1. Cheung A, Webb JG, Wong DR (2009) Transapical transcatheter mitral valve-in-valve implantation in a human. Ann Thorac Surg 87(3): 18-20.

2. Seeburger J, Rinaldi M, Nielson SL (2014) Off- pump transapical implantation of artificial neo-chordae to correct mitral regurgitation: The TACT trial (Transapical artificial chordae Tendinae) proof of concept. J Am Coll Cardiol 63(9): 914-919.

3. Yarboro LT, Kron IL (2016) How I teachmitral valve surgery. Ann Thorac Surg 101(5): 1641-1643.

4. McCarthy JF, Cosgrove DM (1998) Optimizing mitral valve exposure with conventional Left atriotomy. Ann Thorac Surg 65(4): 1161-1162.

5. Zacharias A (1986) Alternative method to improve exposure for difficult mitral valve procedures. Ann Thorac Surg 42(3): 336-337.

6. Legare JF, Buth KJ, Arora RC, Murphy DA, Sullivan JA, et al. (2003) The dome of the left atrium: An alternative approach for mitral valve repair. Eur J Cardiothorac Surg 23(3): 272-276.

7. Nienabar JJ, Glower DD (2006) Mini trans septal versus left atrial approach to the mitral valve: A comparision of outcomes. Ann Thorac Surg 82(3): 834-839.

ISSN: 2574-1241

DOI: 10.26717/BJSTR.2018.09.001867

Kenan Abdurrahman Kara. Biomed J Sci \& Tech Res

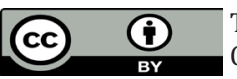

This work is licensed under Creative Commons Attribution 4.0 License

Submission Link: https://biomedres.us/submit-manuscript.php
8. Rezahosseini O, Rezaei M, Ahmadi Tafti SH (2015) Transseptal approach versus left atrial approach to mitral valve: a propensity score matching study. J Teh Univ Heart Ctr 10(4): 188-193.

9. Deloche A, Acar C, Jebara V, Fabiani JN, Carpentier A (1990) Biatrial transseptal approach in case of difficult exposure to themitral valve. Ann Thorac Surg 50(2): 318-319.

10. Ansar T, Ali T, Shahid S, Fatimi S, Murtaza G (2017) Superior septal approach versus left atrial approach for mitral valve replacement: a retrospective cohort study. JPMA: Journal of the Pakistan Medical Association 67(2): 322-326.

11. Gaudino M, Nasso G, Minati A, Salica A, Luciani N, et al. (2003) Early and late arrhythmias in patients in preoperative sinus rhythm submitted to mitral valve surgery through the superior septal approach. Ann Thorac Surg 75(4): 1181-1184.

12. Gaudino M, Alessandrini F, Glieca F, Martinelli L, Santarelli P, et al. (1997) Conventional left atrial versus superior septal approach for mitral valve replacement. Ann Thorac Surg 63(4): 1123-1127.

13. Garcia-Villarreal OA, Gonzalez-Oviedo R, Rodriguez-Gonzalez H, Martinez-Chapa HD (2003) Superior septal approach for mitral valve surgery: A word of caution. Eur J Cardiothorac Surg 24(6): 862-867.

14. Lukac P, Hjortdal VE, Pedersen AK, Mortensen PT, Jensen HK, et al. (2007) Superior transseptal approach to mitral valve is associated with a higher need for pacemaker implantation than the left atrial approach. Ann Thorac Surg 83(1): 77-82.

15. Nienaber JJ, Glower DD (2006) Mini trans septal versus left atrial approach to the mitral valve: A comparison of outcomes. Ann Thorac Surg 82: 834-839.

16. Berreklouw E, Ercan H, Schonberger JP (1991) Combined superior transseptal approach to the left atrium. Ann Thorac Surg 51(2): 293-295.

17. Guiraudon GM, Ofiesh JG, Kaushik R (1991) Extended vertical transatrial septal approach to the mitral valve. Ann Thorac Surg 52(5): 1058-1062.

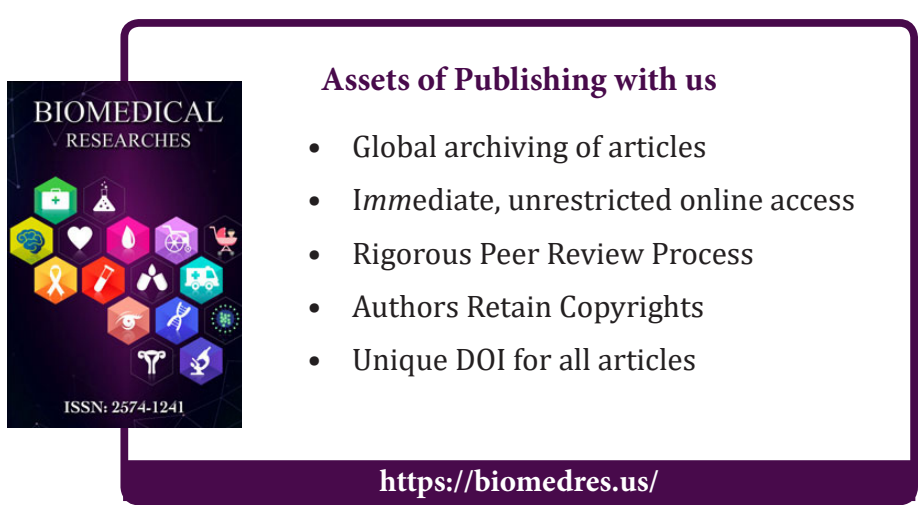

